\title{
Predictive control of multivariable time-delay systems
}

\author{
Kubalcik Marek $^{1, *}$, Bobal Vladimir ${ }^{1}$ \\ ${ }^{1}$ Tomas Bata University in Zlin, Faculty of Applied Informatics, Nam. T. G. Masaryka 5555, 76005 Zlin, Czech Republic
}

\begin{abstract}
In technical practice often occur multivariable processes with time delay. Time-delays are mainly caused by the time required to transport mass, energy or information, but they can also be caused by processing time or accumulation. In a multivariable system each input may influence all system outputs. The design of a controller for such a system must be quite sophisticated if the system is to be controlled adequately. One of the possible approaches to control of multivariable time-delay processes is application of predictive control methods. The paper deals with design of an algorithm for predictive control of multivariable processes with time-delay. The predictive controller is based on the recursive computation of predictions which was extended for the time-delay system. The control of a multivariable system with two steps of time-delay was verified by simulation.
\end{abstract}

\section{Introduction}

Typical technological processes require the simultaneous control of several variables related to one system. Each input may influence all system outputs. The design of a controller for such a system must be quite sophisticated if the system is to be controlled adequately. Simple decentralized PI or PID controllers largely do not yield satisfactory results. There are many different advanced methods of controlling multi-input-multi-output (MIMO) systems. The problem of selecting an appropriate control technique often arises. Perhaps the most popular way of controlling MIMO processes is by designing decoupling compensators to suppress the interactions [1] and the designing multiple SISO controllers [2] . This requires determining how to pair the controlled and manipulated variables. One of the most effective approaches to control of multivariable systems is model predictive control (MPC) [3], [4], [5]. An advantage of model predictive control is that multivariable systems can be handled in a straightforward manner.

In technical practice often occur multivariable processes with time delay.Time-delays are mainly caused by the time required to transport mass, energy or information, but they can also be caused by processing time or accumulation. Typical examples of such processes are e.g. liquid storing tanks, distillation columns or some types of chemical reactors. Time-delay may be defined as the time interval between the start of an event at one point in a system and is resulting action at another point in a system and its resulting action at another point in the system. One older classification of techniques for the compensation of time-delayed processes is introduced in [6], [7] and newer overview of recent advances and open problems it is possible to find in [8]. Processes with time-delay in general are difficult to control using standard feedback controllers. One of the possible approaches to control processes with time delay is predictive control. The predictive control strategy includes a model of the process in the structure of the controller. The first time-delay compensation algorithm was proposed in [9]. This control algorithm known as the Smith Predictor (SP) contained a dynamic model of the time-delay process and it can be considered as the first model predictive algorithm.

When using most of other approaches, the control actions are taken based on past errors. MPC uses also future values of the reference signals. It is essentially based on discrete or sampled models of processes. Computation of appropriate control algorithms is then realized especially in the discrete domain. The basic idea of the generalized predictive control [10], [11] is to use a model of a controlled process to predict a number of future outputs of the process. A trajectory of future manipulated variables is given by solving an optimization problem incorporating a suitable cost function and constraints. Only the first element of the obtained control sequence is applied. The whole procedure is repeated in following sampling period. This principle is known as the receding horizon strategy.

An implementation of a multivariable predictive controller for control of time-delay systems based on a matrix fraction model is described in this paper. A computation of predictions for the case with the timedelay is introduced. The computation is based on a particular model of the controlled system in the form of matrix fraction which is commonly used for description of a range of processes. For the purpose of simplification it was considered equal time-delay in all particular transfer functions of the transfer matrix. The proposed algorithm is then verified by simulation.

\footnotetext{
Corresponding author: kubalcik@fai.utb.cz
} 


\section{Model of the controlled system}

Let us consider a two input - two output system. The two - input/two - output (TITO) processes are the most often encountered multivariable processes in practice and many processes with inputs/outputs beyond two can be treated as several TITO subsystems [12].

A general transfer matrix of a two-input-two-output system with significant cross-coupling between the control loops is expressed as:

$$
\begin{gathered}
\boldsymbol{G}(z)=\left[\begin{array}{ll}
G_{11}(z) & G_{12}(z) \\
G_{21}(z) & G_{22}(z)
\end{array}\right] \\
\boldsymbol{Y}(z)=\boldsymbol{G}(z) \boldsymbol{U}(z)
\end{gathered}
$$

where $\boldsymbol{U}(z)$ and $\boldsymbol{Y}(z)$ are vectors of the manipulated variables and the controlled variables, respectively.

$$
\boldsymbol{U}(z)=\left[u_{1}(z), u_{2}(z)\right]^{T} \quad \boldsymbol{Y}(z)=\left[y_{1}(z), y_{2}(z)\right]^{T}
$$

It may be assumed that the transfer matrix can be transcribed to the following form of the matrix fraction:

$$
\boldsymbol{G}(z)=\boldsymbol{A}^{-1}\left(z^{-1}\right) \boldsymbol{B}\left(z^{-1}\right)=\boldsymbol{B}_{1}\left(z^{-1}\right) \boldsymbol{A}_{1}^{-1}\left(z^{-1}\right)
$$

where the polynomial matrices $\boldsymbol{A} \in R_{22}\left[z^{-1}\right], \boldsymbol{B} \in R_{22}\left[z^{-1}\right]$ are the left coprime factorizations of matrix $\boldsymbol{G}(z)$ and the matrices $\boldsymbol{A}_{1} \in R_{22}\left[z^{-1}\right], \boldsymbol{B}_{1} \in R_{22}\left[z^{-1}\right]$ are the right coprime factorizations of $\boldsymbol{G}(z)$. The model can be also written in the form

$$
\boldsymbol{A}\left(z^{-1}\right) \boldsymbol{Y}(z)=\boldsymbol{B}\left(z^{-1}\right) \boldsymbol{U}(z)
$$

As an example a model with polynomials of second degree was chosen. This model proved to be effective for control of several TITO laboratory processes [13], where controllers based on a model with polynomials of the first degree failed. The model has sixteen parameters. The matrices $\boldsymbol{A}$ and $\boldsymbol{B}$ are defined as follows

$$
\begin{gathered}
A\left(z^{-1}\right)=\left[\begin{array}{cc}
1+a_{1} z^{-1}+a_{2} z^{-2} & a_{3} z^{-1}+a_{4} z^{-2} \\
a_{5} z^{-1}+a_{6} z^{-2} & 1+a_{7} z^{-1}+a_{8} z^{-2}
\end{array}\right] \\
\boldsymbol{B}\left(z^{-1}\right)=\left[\begin{array}{cc}
b_{1} z^{-1}+b_{2} z^{-2} & b_{3} z^{-1}+b_{4} z^{-2} \\
b_{5} z^{-1}+b_{6} z^{-2} & b_{7} z^{-1}+b_{8} z^{-2}
\end{array}\right]
\end{gathered}
$$

A widely used model in general model predictive control is the CARIMA (controller autoregressive integrated moving average) model which we can obtain by adding a disturbance model as

$$
\boldsymbol{A}\left(z^{-1}\right) \boldsymbol{y}(k)=\boldsymbol{B}\left(z^{-1}\right) \boldsymbol{u}(k)+\boldsymbol{C}\left(z^{-1}\right) \boldsymbol{\Delta}^{-1}\left(z^{-1}\right) \boldsymbol{n}(k)
$$

where $\boldsymbol{n}$ is a non-measurable random disturbance that is assumed to have zero mean value and constant covariance and

$$
\Delta\left(z^{-1}\right)=\left[\begin{array}{cc}
1-z^{-1} & 0 \\
0 & 1-z^{-1}
\end{array}\right]
$$

in case of TITO system.

The nominal model with $d$ steps of time-delay is considered as

$$
\boldsymbol{G}(z)=\boldsymbol{A}^{-1}\left(z^{-1}\right) \boldsymbol{B}\left(z^{-1}\right) z^{-d}=\boldsymbol{B}_{1}\left(z^{-1}\right) \boldsymbol{A}_{1}^{-1}\left(z^{-1}\right) z^{-d}
$$

For the purpose of simplification it was considered equal time-delay in all particular transfer functions of the transfer matrix. The CARIMA model for time-delay system then takes the form

$$
\boldsymbol{A}\left(z^{-1}\right) \boldsymbol{y}(k)=z^{-d} \boldsymbol{B}\left(z^{-1}\right) \boldsymbol{u}(k)+\boldsymbol{C}\left(z^{-1}\right) \boldsymbol{\Delta}^{-1}\left(z^{-1}\right) \boldsymbol{n}(k)
$$

\section{Implementation of predictive controller}

The basic idea of MPC is to use a model of a controlled process to predict $N$ future outputs of the process. A trajectory of future manipulated variables is given by solving an optimization problem incorporating a suitable cost function and constraints. Only the first element of the obtained control sequence is applied. The whole procedure is repeated in following sampling period. This principle is known as the receding horizon strategy. The computation of a control law of MPC is based on minimization of the following criterion

$$
J(k)=\sum_{j=1}^{N} \boldsymbol{e}(k+j)^{2}+\lambda \sum_{j=1}^{N_{u}} \Delta \boldsymbol{u}(k+j)^{2}
$$

where $\boldsymbol{e}(k+j)$ is a vector of predicted control errors, $\Delta \boldsymbol{u}(k+j)$ is a vector of future increments of the manipulated variable (for the system with two inputs and two outputs each vector has two elements), $N$ is a length of the prediction horizon, $N_{u}$ is a length of the control horizon and $\lambda$ is a weighting factor of control increments.

A predictor in a vector form is given by

$$
\hat{\boldsymbol{y}}=\boldsymbol{G} \Delta \boldsymbol{u}+\boldsymbol{y}_{0}
$$

where $\hat{\boldsymbol{y}}$ is a vector of system predictions along the horizon of the length $N, \Delta \boldsymbol{u}$ is a vector of control increments, $\boldsymbol{y}_{0}$ is the free response vector. $\boldsymbol{G}$ is a matrix of the dynamics. It is given as

$$
\boldsymbol{G}=\left[\begin{array}{ccccc}
\boldsymbol{G}_{0} & 0 & \cdots & \cdots & 0 \\
\boldsymbol{G}_{1} & \boldsymbol{G}_{0} & 0 & \cdots & 0 \\
\vdots & & \ddots & \ddots & \vdots \\
\vdots & & & \boldsymbol{G}_{0} & 0 \\
\boldsymbol{G}_{N-1} & \cdots & \cdots & \cdots & \boldsymbol{G}_{0}
\end{array}\right]
$$

where sub-matrices $\boldsymbol{G}_{i}$ have dimension $2 \times 2$ and contain values of the step sequence.

The criterion (12) can be written in a general vector form 


$$
J=(\hat{\boldsymbol{y}}-\boldsymbol{w})^{T}(\hat{\boldsymbol{y}}-\boldsymbol{w})+\lambda \Delta \boldsymbol{u}^{T} \Delta \boldsymbol{u}
$$

where $\boldsymbol{w}$ is a vector of the reference trajectory. The criterion can be modified using the expression (15) to

$$
J=2 \boldsymbol{g}^{T} \Delta \boldsymbol{u}+\Delta \boldsymbol{u}^{T} \boldsymbol{H} \Delta \boldsymbol{u}
$$

where the gradient $\boldsymbol{g}$ and the Hess matrix $\boldsymbol{H}$ are defined by following expressions

$$
\begin{gathered}
\boldsymbol{g}^{T}=\boldsymbol{G}^{T}\left(\boldsymbol{y}_{0}-\boldsymbol{w}\right) \\
\boldsymbol{H}=\boldsymbol{G}^{T} \boldsymbol{G}
\end{gathered}
$$

Handling of constraints is one of main advantages of predictive control. General formulation of predictive control with constraints is then as follows

$$
\min _{\Delta \boldsymbol{u}} 2 \boldsymbol{g}^{T} \Delta \boldsymbol{u}+\Delta \boldsymbol{u}^{T} \boldsymbol{H} \Delta \boldsymbol{u}
$$

owing to

$$
\boldsymbol{A} \Delta \boldsymbol{u} \leq \boldsymbol{b}
$$

The inequality (20) expresses the constraints in a compact form.

\section{Computation of predictor}

An important task is computation of predictions for arbitrary prediction and control horizons. Dynamics of most of processes requires horizons of length where it is not possible to compute predictions in a simple straightforward way. Recursive expressions for computation of the free response and the matrix $\boldsymbol{G}$ in each sampling period had to be derived. There are several different ways of deriving the prediction equations for transfer function models. Some papers make use of Diophantine equations to form the prediction equations [14]. In [15] matrix methods are used to compute predictions. We derived a method for recursive computation of both the free response and the matrix of the dynamics.

Computation of the predictor for the time-delay system can be obtained by modification of the predictor for the corresponding system without a time-delay. At first we will consider the TITO system without timedelay and then we will modify the computation of predictions for the time-delay system.

\subsection{TITO system without time-delay}

The difference equation of the CARIMA model without the unknown term can be expressed as:

$$
\begin{aligned}
& y_{1}(k+1)=\left(1-a_{1}\right) y_{1}(k)+\left(a_{1}-a_{2}\right) y_{1}(k-1)+a_{2} y_{1}(k-2)- \\
& -a_{3} y_{2}(k)+\left(a_{3}-a_{4}\right) y_{2}(k-1)+a_{4} y_{2}(k-2)+ \\
& +b_{1} \Delta u_{1}(k)+b_{2} \Delta u_{1}(k-1)+b_{3} \Delta u_{2}(k)+b_{4} \Delta u_{2}(k-1)
\end{aligned}
$$

$$
\begin{aligned}
& y_{2}(k+1)=\left(1-a_{7}\right) y_{2}(k)+\left(a_{7}-a_{8}\right) y_{2}(k-1)+a_{8} y_{2}(k-2)- \\
& -a_{5} y_{1}(k)+\left(a_{5}-a_{6}\right) y_{1}(k-1)+a_{6} y_{1}(k-2)+ \\
& +b_{5} \Delta u_{1}(k)+b_{6} \Delta u_{1}(k-1)+b_{7} \Delta u_{2}(k)+b_{8} \Delta u_{2}(k-1)
\end{aligned}
$$

These equations can be written into a matrix form

$$
\begin{aligned}
& \boldsymbol{y}(k+1)=\boldsymbol{A}_{1} \boldsymbol{y}(k)+\boldsymbol{A}_{2} \boldsymbol{y}(k-1)+\boldsymbol{A}_{3} \boldsymbol{y}(k-2)+ \\
& +\boldsymbol{B}_{1} \Delta \boldsymbol{u}(k)+\boldsymbol{B}_{2} \Delta \boldsymbol{u}(k-1)
\end{aligned}
$$

where

$$
\begin{gathered}
\boldsymbol{A}_{1}=\left[\begin{array}{cc}
1-a_{1} & -a_{3} \\
-a_{5} & 1-a_{7}
\end{array}\right] \boldsymbol{A}_{2}=\left[\begin{array}{ll}
a_{1}-a_{2} & a_{3}-a_{4} \\
a_{5}-a_{6} & a_{7}-a_{8}
\end{array}\right] \\
\boldsymbol{A}_{3}=\left[\begin{array}{ll}
a_{2} & a_{4} \\
a_{6} & a_{8}
\end{array}\right] \\
\boldsymbol{B}_{1}=\left[\begin{array}{ll}
b_{1} & b_{3} \\
b_{5} & b_{7}
\end{array}\right] \boldsymbol{B}_{2}=\left[\begin{array}{ll}
b_{2} & b_{4} \\
b_{6} & b_{8}
\end{array}\right]
\end{gathered}
$$

It was necessary to directly compute three stepsahead predictions in a straightforward way by establishing of previous predictions to later predictions. The model order defines that computation of one stepahead prediction is based on the three past values of the system output.

$$
\begin{aligned}
& \hat{\boldsymbol{y}}(k+1)=\boldsymbol{A}_{1} \boldsymbol{y}(k)+\boldsymbol{A}_{2} \boldsymbol{y}(k-1)+\boldsymbol{A}_{3} \boldsymbol{y}(k-2)+ \\
& +\boldsymbol{B}_{1} \Delta \boldsymbol{u}(k)+\boldsymbol{B}_{2} \Delta \boldsymbol{u}(k-1) \\
& \quad \hat{\boldsymbol{y}}(k+2)=\boldsymbol{A}_{1} \boldsymbol{y}(k+1)+\boldsymbol{A}_{2} \boldsymbol{y}(k)+\boldsymbol{A}_{3} \boldsymbol{y}(k-1)+ \\
& \quad+\boldsymbol{B}_{1} \Delta \boldsymbol{u}(k+1)+\boldsymbol{B}_{2} \Delta \boldsymbol{u}(k) \\
& \quad \hat{\boldsymbol{y}}(k+3)=\boldsymbol{A}_{1} \boldsymbol{y}(k+2)+\boldsymbol{A}_{2} \boldsymbol{y}(k+1)+\boldsymbol{A}_{3} \boldsymbol{y}(k)+ \\
& \quad+\boldsymbol{B}_{1} \Delta \boldsymbol{u}(k+2)+\boldsymbol{B}_{2} \Delta \boldsymbol{u}(k+1)
\end{aligned}
$$

The three steps ahead predictions can be expressed using (13) as follows

$$
\left[\begin{array}{l}
\hat{\boldsymbol{y}}(k+1) \\
\hat{\boldsymbol{y}}(k+2) \\
\hat{\boldsymbol{y}}(k+3)
\end{array}\right]=\boldsymbol{G} \Delta \boldsymbol{u}+\boldsymbol{y}_{0}
$$

It is possible to divide computation of the predictions to recursion of the free response and recursion of the matrix of the dynamics. The free response vector predictions can be expressed as:

$$
\begin{aligned}
& \boldsymbol{y}_{0}=\left[\begin{array}{ll}
p_{11} & p_{12} \\
p_{21} & q_{22} \\
\hline p_{31} & p_{32} \\
p_{41} & p_{42} \\
\hline p_{51} & p_{52} \\
p_{61} & p_{62}
\end{array}\right]\left[\begin{array}{ll}
\Delta u_{1}(k-1) \\
\Delta u_{2}(k-1)
\end{array}\right]+\left[\begin{array}{ll|ll|ll}
q_{11} & q_{12} & q_{13} & q_{14} & q_{15} & q_{16} \\
q_{21} & q_{22} & q_{23} & q_{24} & q_{25} & q_{26} \\
\hline q_{31} & q_{32} & q_{33} & q_{34} & q_{35} & q_{36} \\
q_{41} & q_{42} & q_{43} & q_{44} & q_{45} & q_{46} \\
\hline q_{51} & q_{52} & q_{53} & q_{54} & q_{55} & q_{56} \\
q_{61} & q_{62} & q_{63} & q_{64} & q_{65} & q_{66}
\end{array}\right]\left[\begin{array}{c}
y_{1}(k) \\
y_{2}(k) \\
y_{1}(k-1) \\
y_{2}(k-1) \\
y_{1}(k-2) \\
y_{2}(k-2)
\end{array}\right]= \\
& =\left[\begin{array}{l}
\boldsymbol{P}_{1} \\
\boldsymbol{P}_{2} \\
\boldsymbol{P}_{3}
\end{array}\right] \Delta \boldsymbol{u}(k-1)+\left[\begin{array}{lll}
\boldsymbol{Q}_{11} & \boldsymbol{Q}_{12} & \boldsymbol{Q}_{13} \\
\boldsymbol{Q}_{21} & \boldsymbol{Q}_{22} & \boldsymbol{Q}_{23} \\
\boldsymbol{Q}_{31} & \boldsymbol{Q}_{32} & \boldsymbol{Q}_{33}
\end{array}\right]\left[\begin{array}{c}
\boldsymbol{y}(k) \\
\boldsymbol{y}(k-1) \\
\boldsymbol{y}(k-2)
\end{array}\right]=\boldsymbol{P} \Delta \boldsymbol{u}(k-1)+\boldsymbol{Q}\left[\begin{array}{c}
\boldsymbol{y}(k) \\
\boldsymbol{y}(k-1) \\
\boldsymbol{y}(k-2)
\end{array}\right]
\end{aligned}
$$

The coefficients of the matrices $\boldsymbol{P}$ and $\boldsymbol{Q}$ for further predictions are computed recursively. Based on the three 
previous predictions it is repeatedly computed the next row of the matrices $\boldsymbol{P}$ and $\boldsymbol{Q}$ in the following way:

$$
\begin{gathered}
\boldsymbol{P}_{4}=\left[\begin{array}{ll}
p_{71} & p_{72} \\
p_{81} & p_{82}
\end{array}\right]=\boldsymbol{A}_{1} \boldsymbol{P}_{31}+\boldsymbol{A}_{2} \boldsymbol{P}_{21}+\boldsymbol{A}_{3} \boldsymbol{P}_{11} \\
\boldsymbol{Q}_{41}=\left[\begin{array}{ll}
q_{71} & q_{72} \\
q_{81} & q_{82}
\end{array}\right]=\boldsymbol{A}_{1} \boldsymbol{Q}_{31}+\boldsymbol{A}_{2} \boldsymbol{Q}_{21}+\boldsymbol{A}_{3} \boldsymbol{Q}_{11} \\
\boldsymbol{Q}_{42}=\left[\begin{array}{ll}
q_{73} & q_{74} \\
q_{83} & q_{84}
\end{array}\right]=\boldsymbol{A}_{1} \boldsymbol{Q}_{32}+\boldsymbol{A}_{2} \boldsymbol{Q}_{22}+\boldsymbol{A}_{3} \boldsymbol{Q}_{12} \\
\boldsymbol{Q}_{43}=\left[\begin{array}{ll}
q_{75} & q_{76} \\
q_{85} & q_{86}
\end{array}\right]=\boldsymbol{A}_{1} \boldsymbol{Q}_{33}+\boldsymbol{A}_{2} \boldsymbol{Q}_{23}+\boldsymbol{A}_{3} \boldsymbol{Q}_{13}
\end{gathered}
$$

The recursion of the matrix $\boldsymbol{G}$ is similar. The next element of the first column is repeatedly computed and the remaining columns are shifted. This procedure is performed repeatedly until the prediction horizon is achieved. If the control horizon is lower than the prediction horizon a number of columns in the matrix is reduced. The technique is apparent from the equations (32) and (33).

$$
\begin{gathered}
\boldsymbol{G} \Delta \boldsymbol{u}=\left[\begin{array}{ll|lc}
g_{11} & g_{12} & 0 & 0 \\
g_{21} & g_{22} & 0 & 0 \\
\hline g_{31} & g_{32} & g_{11} & g_{12} \\
g_{41} & g_{42} & g_{21} & g_{22} \\
\hline g_{51} & g_{52} & g_{31} & g_{32} \\
g_{61} & g_{62} & g_{41} & g_{42}
\end{array}\right]\left[\begin{array}{c}
\Delta u_{1}(k) \\
\Delta u_{2}(k) \\
\Delta u_{1}(k+1) \\
\Delta u_{2}(k+1)
\end{array}\right]= \\
=\left[\begin{array}{ll}
\boldsymbol{G}_{1} & 0 \\
\boldsymbol{G}_{2} & \boldsymbol{G}_{1} \\
\boldsymbol{G}_{3} & \boldsymbol{G}_{2}
\end{array}\right]\left[\begin{array}{c}
\Delta \boldsymbol{u}(k) \\
\Delta \boldsymbol{u}(k+1)
\end{array}\right] \\
\boldsymbol{G}_{4}=\left[\begin{array}{ll}
g_{71} & g_{72} \\
g_{81} & g_{82}
\end{array}\right]=\boldsymbol{A}_{1} \boldsymbol{G}_{3}+\boldsymbol{A}_{2} \boldsymbol{G}_{2}+\boldsymbol{A}_{3} \boldsymbol{G}_{1}
\end{gathered}
$$
form

The predictions can be written in a compact matrix

$$
\begin{aligned}
& \hat{\boldsymbol{y}}(k+j)=\boldsymbol{G} \Delta \boldsymbol{u}(k+j-1)+\boldsymbol{P} \Delta \boldsymbol{u}(k-1)+\boldsymbol{Q} \boldsymbol{y}(k-j+1) \\
& j \leq N
\end{aligned}
$$

\subsection{TITO system with time-delay}

The nominal model with two steps time-delay is considered as

$$
\boldsymbol{G}(z)=\boldsymbol{A}^{-1}\left(z^{-1}\right) \boldsymbol{B}\left(z^{-1}\right) z^{-2}=\boldsymbol{B}_{1}\left(z^{-1}\right) \boldsymbol{A}_{1}^{-1}\left(z^{-1}\right) z^{-2}
$$

The CARIMA model for time-delay system takes the form (11) In our case $d$ is equal to 2. In order to compute the control action it is necessary to determine the predictions from $d+1(2+1$ in our case $)$ to $d+N_{2}\left(2+N_{2}\right)$.

$$
\left[\begin{array}{l}
\hat{\boldsymbol{y}}(k+3) \\
\hat{\boldsymbol{y}}(k+4) \\
\hat{\boldsymbol{y}}(k+5)
\end{array}\right]=\boldsymbol{G \Delta} \boldsymbol{u}+\boldsymbol{y}_{0}
$$

The computation of free response is then modified to $\boldsymbol{y}_{0}=\left[\begin{array}{ll|ll|ll}g_{31} & g_{32} & g_{51} & g_{52} & p_{51} & p_{52} \\ g_{41} & g_{42} & g_{61} & g_{62} & p_{61} & p_{62} \\ \hline g_{51} & g_{52} & g_{71} & g_{72} & p_{71} & p_{72} \\ g_{61} & g_{62} & g_{81} & g_{82} & p_{81} & p_{82} \\ \hline g_{71} & g_{72} & g_{91} & g_{92} & p_{91} & p_{92} \\ g_{81} & g_{82} & g_{101} & g_{102} & p_{101} & p_{102}\end{array}\right]\left[\begin{array}{c}\Delta u_{1}(k-1) \\ \Delta u_{2}(k-1) \\ \Delta u_{1}(k-2) \\ \Delta u_{2}(k-2) \\ \Delta u_{1}(k-3) \\ \Delta u_{2}(k-3)\end{array}\right]+$ $+\left[\begin{array}{cc|cc|cc}q_{51} & q_{52} & q_{53} & q_{54} & q_{55} & q_{56} \\ q_{61} & q_{62} & q_{63} & q_{64} & q_{65} & q_{66} \\ \hline q_{71} & q_{72} & q_{73} & q_{34} & q_{75} & q_{76} \\ q_{81} & q_{82} & q_{83} & q_{44} & q_{85} & q_{86} \\ \hline q_{91} & q_{92} & q_{93} & q_{54} & q_{95} & q_{96} \\ q_{101} & q_{102} & q_{103} & q_{64} & q_{105} & q_{106}\end{array}\right]\left[\begin{array}{c}y_{1}(k) \\ y_{2}(k) \\ y_{1}(k-1) \\ y_{2}(k-1) \\ y_{1}(k-2) \\ y_{2}(k-2)\end{array}\right]=$ $=\left[\begin{array}{lll}\boldsymbol{G}_{2} & \boldsymbol{G}_{3} & \boldsymbol{P}_{3} \\ \boldsymbol{G}_{3} & \boldsymbol{G}_{4} & \boldsymbol{P}_{4} \\ \boldsymbol{G}_{4} & \boldsymbol{G}_{5} & \boldsymbol{P}_{5}\end{array}\right]\left[\begin{array}{c}\Delta \boldsymbol{u}(k-1) \\ \Delta \boldsymbol{u}(k-2) \\ \Delta \boldsymbol{u}(k-3)\end{array}\right]+\left[\begin{array}{lll}\boldsymbol{Q}_{31} & \boldsymbol{Q}_{32} & \boldsymbol{Q}_{33} \\ \boldsymbol{Q}_{41} & \boldsymbol{Q}_{42} & \boldsymbol{Q}_{43} \\ \boldsymbol{Q}_{51} & \boldsymbol{Q}_{52} & \boldsymbol{Q}_{53}\end{array}\right]\left[\begin{array}{c}\boldsymbol{y}(k) \\ \boldsymbol{y}(k-1) \\ \boldsymbol{y}(k-2)\end{array}\right]=$ $=\boldsymbol{P}_{1}\left[\begin{array}{c}\Delta \boldsymbol{u}(k-1) \\ \Delta \boldsymbol{u}(k-2) \\ \Delta \boldsymbol{u}(k-3)\end{array}\right]+\boldsymbol{Q}_{1}\left[\begin{array}{c}\boldsymbol{y}(k) \\ \boldsymbol{y}(k-1) \\ \boldsymbol{y}(k-2)\end{array}\right]$

The computation of forced response (32) remains the same. Recursive computation of the matrices is analogical to the recursive computation described in section 4.1.

\subsection{Modified predictor for arbitrary time-delay}

The predictor modified for an arbitrary time -delay is then given as follows.

$$
\left[\begin{array}{l}
\hat{\boldsymbol{y}}(k+1+d) \\
\hat{\boldsymbol{y}}(k+2+d) \\
\hat{\boldsymbol{y}}(k+3+d)
\end{array}\right]=\boldsymbol{G} \Delta \boldsymbol{u}+\boldsymbol{y}_{0}
$$




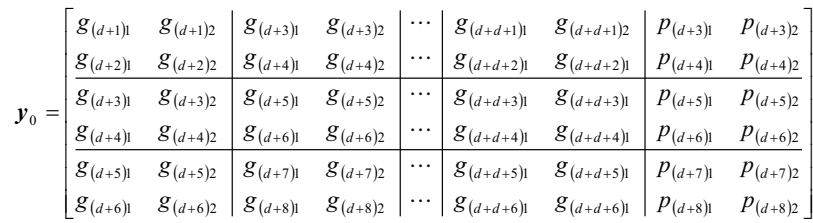

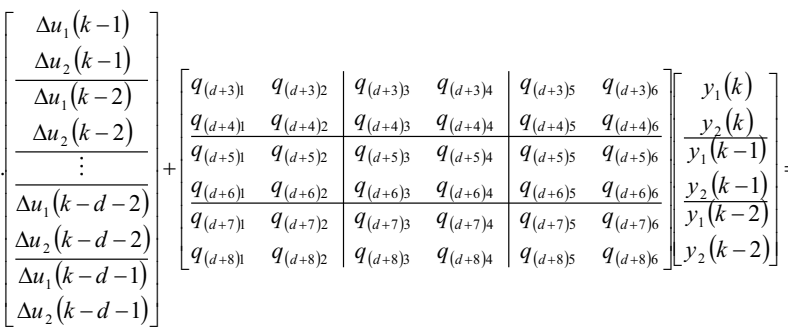

$$
\begin{aligned}
& =\left[\begin{array}{ccccc}
\boldsymbol{G}_{d} & \boldsymbol{G}_{d+1} & \cdots & \boldsymbol{G}_{d+d-1} & \boldsymbol{P}_{d+1} \\
\boldsymbol{G}_{d+1} & \boldsymbol{G}_{d+2} & \cdots & \boldsymbol{G}_{d+d} & \boldsymbol{P}_{d+2} \\
\boldsymbol{G}_{d+2} & \boldsymbol{G}_{d+3} & \cdots & \boldsymbol{G}_{d+d+1} & \boldsymbol{P}_{d+3}
\end{array}\right]\left[\begin{array}{c}
\Delta \boldsymbol{u}(k-1) \\
\Delta \boldsymbol{u}(k-2) \\
\vdots \\
\Delta \boldsymbol{u}(k-d-2) \\
\Delta \boldsymbol{u}(k-d-1)
\end{array}\right]+ \\
& +\left[\begin{array}{lll}
\boldsymbol{Q}_{(d+1) !} & \boldsymbol{Q}_{(d+1) 2} & \boldsymbol{Q}_{(d+1) 3} \\
\boldsymbol{Q}_{(d+2) 1} & \boldsymbol{Q}_{(d+2) 2} & \boldsymbol{Q}_{(d+1) 3}
\end{array}\right]\left[\begin{array}{c}
\boldsymbol{y}(k) \\
\boldsymbol{y}(k-1) \\
\boldsymbol{Q}_{(d+3)}
\end{array}\right. \\
& {\left[\begin{array}{lll}
\boldsymbol{Q}_{(d+3) 1} & \boldsymbol{Q}_{(d+3) 2} & \boldsymbol{Q}_{(d+1) 3}
\end{array}\right]\left[\begin{array}{ll}
\boldsymbol{y}(k-2) \\
\boldsymbol{Q}_{(d+2)}
\end{array}\right]} \\
& =\boldsymbol{P}_{2}\left[\begin{array}{c}
\Delta \boldsymbol{u}(k-1) \\
\Delta \boldsymbol{u}(k-2) \\
\vdots \\
\Delta \boldsymbol{u}(k-d-2) \\
\Delta \boldsymbol{u}(k-d-1)
\end{array}\right]+\boldsymbol{Q}_{2}\left[\begin{array}{c}
\boldsymbol{y}(k) \\
\boldsymbol{y}(k-1) \\
\boldsymbol{y}(k-2)
\end{array}\right]
\end{aligned}
$$

The computation of the forced response is again given by equation (32)

\section{Simulation example}

As simulation example was chosen a system with two steps of time-delay

$$
\boldsymbol{G}(z)=\boldsymbol{A}^{-1}\left(z^{-1}\right) \boldsymbol{B}\left(z^{-1}\right) z^{-2}
$$

described by polynomial matrices (41) -(42)

$A\left(z^{-1}\right)=\left[\begin{array}{cc}1-0.5827 z^{-1}+0.1745 z^{-2} & -0.0220 z^{-1}+0.1797 z^{-2} \\ 0.0167 z^{-1}-0.0886 z^{-2} & 1-0.4564 z^{-1}-0.0830 z^{-2}\end{array}\right]$

$$
\boldsymbol{B}\left(z^{-1}\right)=\left[\begin{array}{cc}
-0.0035 z^{-1}+0.0955 z^{-2} & 0.1484 z^{-1}+0.2197 z^{-2} \\
0.2783 z^{-1}+0.3107 z^{-2} & -0.0371 z^{-1}-0.3489 z^{-2}
\end{array}\right]
$$

Control responses are in figures 1 and 2.

The tuning parameters that are lengths of the prediction and control horizons and the weighting coefficient $\lambda$ were tuned experimentally. There is a lack of clear theory relating to the closed loop behaviour to design parameters. The length of the prediction horizon was set to $N=10$. The length of the control horizon was also set to $N_{u}=10$. The coefficient $\lambda$ was taken as equal to 0,5 .
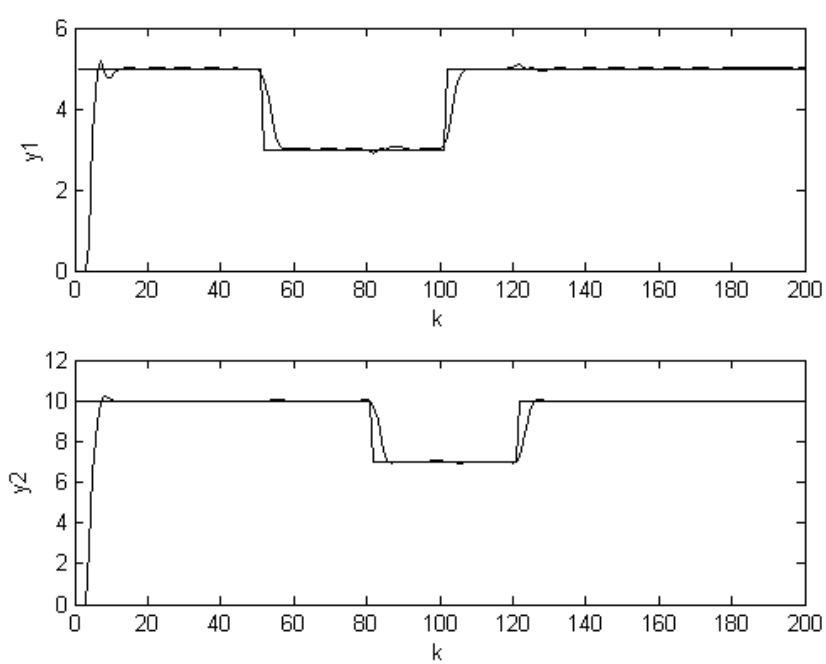

Fig. 1. Simulation results - controlled variable.
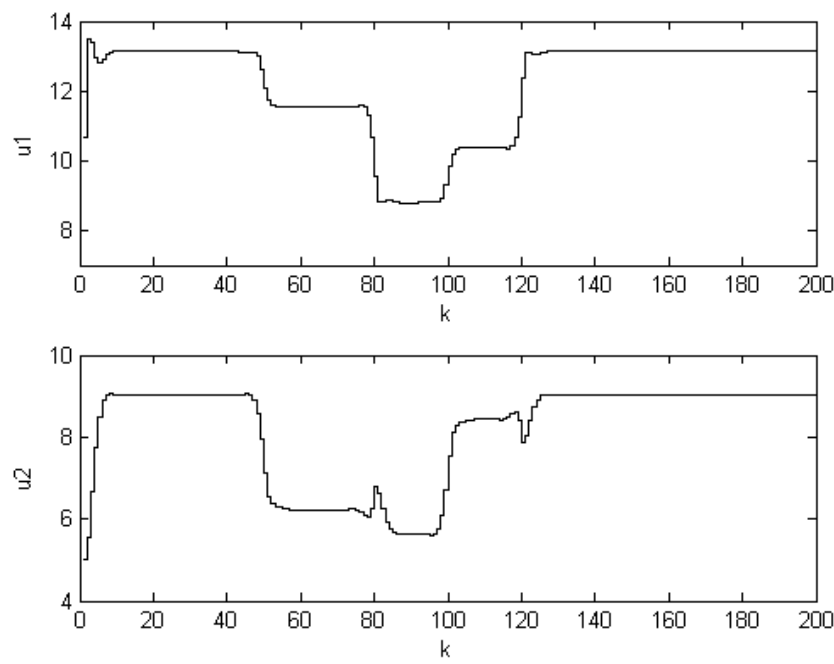

Fig. 2. Simulation results - manipulated variable.

\section{Conclusions}

The algorithm for control of the multivariable time-delay systems based on model predictive control was designed. The predictive controller is based on the recursive computation of predictions by direct use of the CARIMA model. The computation of predictions was extended for the time-delay system. The control of a multivariable system with two steps of time-delay was verified by simulation. The simulation verification provided good control results. Asymptotic tracking of the reference signal was achieved. The control was rather sensitive to tuning parameters. Experimental tuning of the controller was quite complicated.

\section{References}

1. P.R. Krishnawamy, et al., Ind. Eng. Chem. Res., 30, 662 (1991)

2. W. L. Luyben, Ing. Eng. Chem. Process Des. Dev., 25, 654 (1986) 
3. E.F. Camacho, C. Bordons, Model Predictive Control (Springer-Verlag, London, 2004)

4. M. Morari, J.H. Lee, Computers and Chemical Engineering, 23, 667 (1999)

5. J. Mikleš, M. Fikar, Process Modelling, Optimisation and Control (Berlin: Springer-Verlag, 2008)

6. A. O'Dwyer, Proc. of the $3^{\text {rd }}$ IMACS/IEE International Multiconference on Circuits, Systems, Communications and Computers, 176, Athens, Greece (1999)

7. A. O'Dwyer, Proc. of the $3^{\text {rd }}$ IMACS/IEE International Multiconference on Circuits, Systems, Communications and Computers, 187, Athens, Greece (1999)

8. J. P. Richard, Automatica, 39, 1667 (2003)

9. O. J. Smith, Chem. Eng. Progress, 53, 217, (1957)

10. D. W. Clarke, C. Mohtadi, P. S. Tuffs, Automatica, 23,137 (1987)

11. D. W. Clarke, C. Mohtadi, P. S. Tuffs, Automatica, 23,149 (1987)

12. F. G. Shinskey, Process Control System (McGrawHill. New York, 1996)

13. M. Kubalčík, V. Bobál, IMechE Part I: J. Systems and Control Engineering, 220(I7), 641 (2006)

14. W.H. Kwon, H. Choj, D.G. Byun, S. Noh, Automatica, 28, 1235, (1992)

15. J.A. Rossiter, Model Based Predictive Control: a Practical Approach (CRC Press, 2003) 